see that $d=0$. Thus $\left(c_{14}+c_{24}\right) I_{1}=0 . \quad$ Apply $x_{2}=x_{2}^{\prime}+x_{1}^{\prime}$, whence

$$
c_{13}^{\prime}=c_{13}+c_{23}, \quad c_{14}^{\prime}=c_{14}+c_{24}, \quad b_{1}^{\prime}=b_{1}+b_{2}+c_{12} .
$$

Then $c_{14} I_{1}=0$. Hence every $c_{i j} I_{1}=0, I_{1}=l A_{4}$. But $I_{1}$ is free of $A_{4}$. Hence $I_{1}=0, I=0, S=0$.

Theorem. Every linear covariant of $q_{4}$ is a linear function of $L, A_{4} L, K L$.

Next, let $\omega>1$. After subtracting from $C$ a constant multiple of $q_{4} L^{\omega-2}$, whose leader is $b_{4} u$, we have $d=0$ in $S$. Express $S_{1}$ as a polynomial in $c_{12}, c_{13}, b_{1}$, and call $p$ the coefficient of their product. The coefficient of $c_{12} c_{13}$ in $S_{1}^{\prime}-S_{1}=S$, found from (1), is $p\left(b_{4}+c_{14}\right)$, and hence vanishes if $b_{4}=c_{14}$; while $S$ itself vanishes if also $c_{24}=c_{34}=0$. Applying these two conditions to $S=I+b_{4} I_{1}$, we find that

$$
S=\left(b_{4}+1\right) k(n+m K), \quad n, m \text { constants. }
$$

Several tests failed to exclude this leader. Whether or not there are covariants with such a leader $S$ is not discussed here.

In this connection, note the covariant

$$
\Sigma c_{i j}\left(x_{i} x_{j}^{2 r}+x_{i}^{2 r} x_{j}\right) \quad(i, j=1, \cdots, 4 ; i<j),
$$

obtained by replacing the variables in the polar of $(x)$ with respect to $q_{4}$ by $x_{k}^{2 r}(k=1, \cdots, 4)$.

6 . By means of the corollary in $\S 4$, and transformation (1), we readily obtain the

THEOREM. Every quadratic covariant of $q_{4}$ is a linear function of $L^{2}, K L^{2}, I q_{4}$, where $I$ is an invariant.

UNiversity of Chicago, June, 1914.

\title{
THE CONVERSE OF THE HEINE-BOREL THEOREM IN A RIESZ DOMAIN.
}

BY DR. E. W. CHITTENDEN.

(Read before the American Mathematical Society, April 11, 1914.)

IN various generalized forms of the Heine-Borel theorem*

* Cf. M. Fréchet, "Sur quelques points du calcul fonctionnel," Rendiconti del Circolo Matematico di Palermo, vol. 22 (1906), p. 26; and T. H. Hildebrandt, "A contribution to the foundations of Fréchet's calcul fonctionnel," Amer. Jour. of Mathematics, vol. 34 (1912), p. 282. 
for a class $\mathfrak{Q}$ of elements $q$, the condition that $\mathfrak{Q}$ be extremal* appears as a necessary condition. It is the purpose of the present paper to show that this condition is necessary in any domain for which a Fréchet limit is defined; and furthermore, that if the property extremal be replaced by the property selfcompact (a class is self-compact if its every infinite subclass has a limiting element in the class), more general domains may be considered,-notably; those of F. Riesz $\dagger$ for which limiting element is the fundamental relation, and those of T. H. Hildebrandt $\ddagger$ for which a limit of a sequence need not be unique. $\S$

\section{Self-Compact Classes in a Riesz Domain.}

A Riesz domain is formed by a class $\mathfrak{B}$ which admits a relation "limiting element" between single elements and subclasses $\mathfrak{I}$ of $\mathfrak{P}$ with the following properties:

(1) If $\mathfrak{I}$ has a limiting element in $\mathfrak{B}, \mathfrak{I}$ contains at least two elements.

(2) If $p$ is a limiting element of $\mathfrak{I}, p$ is a limiting element of any class containing $\mathfrak{T}$.

(3) If $p$ is a limiting element of $\mathfrak{T}$, and $\mathfrak{T}$ is divided into classes $\mathfrak{I}_{1}, \mathfrak{I}_{2}$, then $p$ is a limiting element of $\mathfrak{I}_{1}$ or $\mathfrak{I}_{2} . \|$

In a Riesz domain an element $p$ is interior $(\Re) \pi$ to $\subseteq$ in case $\mathfrak{S}$ contains $p$ and a single element (distinct from $p$ ) of every class $\mathfrak{I}$ of $\Re$ which has $p$ for a limiting element.

A family [ऽ] of classes $\subseteq$ is an extended enclosure of a class $\mathfrak{Q}$ if for every element $q$ of $\mathfrak{Q}$ there exists an $\mathfrak{S}_{q}$ of $\subseteq$ such that $q$ is interior $(\mathfrak{Q})$ to $\subseteq$.

* Cf. Fréchet, loc. cit., p. 7.

$\dagger$ "Stetigkeitsbegriff und abstrakte Mengenlehre," Atti del IV Congresso Internazionale dei Matematiche, Roma, 1908, vol. 2, pp. 18-24.

$\ddagger$ Loc. cit., p. 241.

$\S$ It is desired to call attention to the fact that it is self-compactness rather than extremality which is essential in many theorems of the calcul fonctionnel of Frechet, and that by the use of this concept most of the theorems of Fréchet which involve the hypothesis of extremality may be extended to domains for which the limit relation is not subject to the uniqueness condition. This has been done to a large extent for classes which admit a development $\Delta$ in a paper presented to the American Mathematical Society in 1913 by Professor Pitcher and Dr. Chittenden, which is not yet published.

II The three properties just given are equivalent to the ones stated by F. Riesz in his Rome paper already cited. That $\mathfrak{T}$ contains an infinity of elements if it has a limiting element is a consequence of (1) and (3). This is established by successive removals of a single element from $\mathfrak{T}$. There always must remain two at least.

$\llbracket$ Read: interior to $\subseteq$ relative to $\Re$. This relativity feature was introduced by T. H. Hildebrandt, loc. cit., p. 268 (10). 
A class $\mathfrak{Q}$ has the Heine-Borel property if every family [S] which is an extended enclosure of $\mathfrak{Q}$ contains a finite subfamily with the same property.

Theorem I. If a class $\mathfrak{O}$ in a Riesz domain has the HeineBorel property, $\mathfrak{D}$ is self-compact.

We have to show that every infinite subclass of $\mathfrak{Q}$ has a limiting element in $\mathfrak{Q}$. Suppose there exists an infinite subclass $\Re$ of $\mathfrak{Q}$ with no limiting element in $\mathfrak{Q}$. From condition (2) it follows that $\Re$ contains a sequence $\left\{q_{n}\right\}$ of distinct elements with no limiting element in $\mathfrak{Q}$. We define $\left[\widetilde{\Im}_{n}\right]$, an

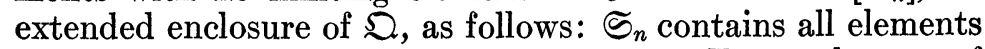
of $\mathfrak{Q}$ except the elements $q_{n+1}, q_{n+2}, \ldots$. Every element of $\mathfrak{Q}$ is contained in some $\widetilde{S}_{n}$. Let $q$ be any element of $\mathfrak{Q}$ and $\mathfrak{I}$ any subclass of $\mathfrak{O}$ which has $q$ for a limiting element. Let $\mathfrak{I}_{1}$ denote the elements of $\mathfrak{I}$ which are in the sequence $\left\{q_{n}\right\}, \mathfrak{I}_{2}$ the remaining elements of $\mathfrak{T}$. By (2) $q$ is not a limiting element of $\mathfrak{T}_{1}$, and by (3) therefore, $q$ is a limiting element of $\mathfrak{I}_{2}$. But $\mathfrak{I}_{2}$ is contained in every $\mathfrak{S}_{n}$. Hence $\left[\mathfrak{S}_{n}\right]$ is an extended enclosure of $\mathfrak{Q}$, such that no finite subfamily of $\left[\Im_{n}\right]$ contains all the elements of $\mathfrak{\Omega}$. This is contrary to hypothesis, and the proposition is proved.*

2. Limiting Element Defined in Terms of Limit. Systems $(\mathfrak{P} ; L)$.

Following T. H. Hildebrandt, $\uparrow$ we denote by $L$ (limit) any relation between a sequence of elements and a single element. If a relation $L$ holds between a sequence $\left\{p_{n}\right\}$ and an element $p$, we say $p$ is a limit of $\left\{p_{n}\right\}$ and write

$$
p=L_{n} p_{n}
$$

A class $\mathfrak{B}$ and a relation $L$ defined for that class form a system $(\mathfrak{P} ; L)$.

The following properties are fundamental in the theory of the relation $L$ :

(1) If a sequence has a limit, the limit is unique.

(2) If a sequence has a limit, its every subsequence, taken in the same order, has the same limit.

(3) The identical sequence $\left\{p_{n}\right\}, p_{n}=p(n)$, has $p$ for a limit. A relation $L$ having a property $i(i=1,2,3,12,13$, $23,123)$ will be denoted by $L^{i}$. An $L^{123}$ is a Fréchet limit. $\ddagger$

* If $\mathfrak{Q}$ is finite it is compact in a vacuous sense. Every finite class has the Heine-Borel property.

$\dagger$ Loc. cit., p. 241.

$\ddagger$ Loc. cit., pp. 6-7. 
In a system $(\mathfrak{B} ; L), p$ is a limiting element of class $\mathfrak{T}$ if $\mathfrak{T}$ contains a sequence $\left\{p_{n}\right\}$ of distinct elements having $p$ for a limit.

The following theorem follows readily from this definition of limiting element.

Theorem II. In a system ( $\left.\mathfrak{B} ; L^{2}\right), \mathfrak{B}$ is a Riesz domain.

As an immediate consequence of theorems I and II we have:

Theorem III. In a system ( $\mathfrak{P} ; L^{2}$ ), every class $\mathfrak{Q}$ which has the Heine-Borel property is self-compact.

We may extend the result of Theorem III with the aid of the following lemma.

Lemma. In a system $\left(\mathfrak{B} ; L^{12}\right)$, every self-compact class $\mathfrak{Q}$ is extremal.

$\mathfrak{Q}$ is compact by hypothesis. We have to show that $\mathfrak{Q}$ is closed. That is, if $p$ is a limiting element of $\mathfrak{T}$, a subclass of $\mathfrak{Q}$, then $p$ belongs to $\mathfrak{Q}$. If $p$ is a limiting element of $\mathfrak{I}$ then a sequence $\left\{q_{n}\right\}$ of distinct elements of $\mathfrak{T}$ exists which has $p$ for a limit. As an infinite class $\left\{q_{n}\right\}$ has a limiting element $q$ in $\mathfrak{Q}$ because $\mathfrak{Q}$ is self-compact. $\left\{q_{n}\right\}$ therefore contains a subsequence $\left\{q_{n_{k}}\right\}$ of distinct elements with limit $q . \quad\left\{q_{n_{k}}\right\}$ contains a subsequence $\left\{q_{n_{k_{m}}}\right\}$ whose order is the same as that of both $\left\{q_{n}\right\}$ and $\left\{q_{n_{k}}\right\}$. Therefore by $L^{2}, q=L_{m} q_{n_{k_{m}}}$ and $p=L_{m} q_{n_{k_{m}}}$. By $L^{1}, q$ and $p$ are the same and therefore $p$ belongs to $\mathfrak{Q}$; which was to be shown. We have from Theorem III and the lemma

Theorem IV. In a system $\left(\mathfrak{B} ; L^{12}\right)$, every class $\mathfrak{Q}$ which has the Heine-Borel property is extremal.

It will be noticed that the character of the result in Theorem IV is determined by the definition of interior given in $\$ 1$ for Riesz domains. We will show that in a system $\left(\mathfrak{P} ; L^{2}\right)$ this definition is (except for relativity) equivalent to the definition used (implicitly) by Fréchet in the theorem cited at the beginning of this paper. It will follow that Theorem IV includes as a special case the result of Fréchet.

The definition of interior used by Fréchet (except for relativity) is equivalent to the following: $q$ is interior $(\Re)$ to $\subseteq$ if $\subseteq$ contains $q$ and every sequence $\left\{r_{n}\right\}$ of distinct elements of $\Re$ which has $q$ for a limit is ultimately* contained in $\widetilde{S}$. in $S$.

* In the sense: there exists $n_{0}$ such that $n \geqq n_{0}$ implies $r_{n}$ is contained 
If now $p$ is Fréchet interior to $\mathfrak{S}$ and is a limiting element of $\mathfrak{T}$ a subclass of $\Re$, then, by definition of Fréchet interior, limiting element, and $L^{2}, \mathfrak{S}$ contains an infinity of elements of $\mathfrak{T}$. Therefore $p$ is interior to $\subseteq$ in the sense of $\S 1$. Furthermore if $p$ is interior $(\Re)$ to $\subseteq$ in the sense of $\S 1$, then $\subseteq$ contains an element $q$ (distinct from $\mathfrak{B}$ ) of every subclass $\mathfrak{T}$ of $\mathfrak{R}$ for which $p$ is a limiting element. Then if $p=L_{n} r_{n}$ (distinct) $p$ is a limiting element of the class $\left[r_{n}\right]$. Hence $\mathfrak{S}$ contains $r_{n_{1}}$ distinct from $p$. Since $p$ is a limiting element of the class obtained from $\left[r_{n}\right]$ by removing $r_{n_{1}}\left(L^{2}\right)$ it is evident that at most a finite number of elements of $\left[r_{n}\right]$ are not in $\mathfrak{S}$. Therefore $\left[r_{n}\right]$ is ultimately contained in $\mathfrak{S}$.

T. H. Hildebrandt* has given a definition of interior $(\Re)$ which becomes equivalent to the Fréchet interior $(\Re)$ for systems $\left(\mathfrak{B} ; L^{123}\right)$. This definition omits the condition that the sequence $\left\{r_{n}\right\}$ consist of distinct elements. If then $p=L_{n} r_{n}$ and $r_{n_{0}}$ is repeated infinitely often, in a system $\left(\mathfrak{P} ; L^{123}\right), r_{n_{0}}=p$. That $r_{n_{0}}$ is contained in any class $\mathbb{S}$ to which $p$ is Fréchet interior $(\Re)$ is evident. A restatement of Theorem IV for systems $\left(\mathfrak{P} ; L^{123}\right)$ gives us a generalization of a theorem of Hildebrandt. $\dagger$

Urbana, Ill.

October 28, 1914.

\title{
COMPLETE EXISTENTIAL THEORY OF SHEFFER'S POSTULATES FOR BOOLEAN ALGEBRAS.
}

\author{
BY PROFESSOR L. L. DINES.
}

(Read before the American Mathematical Society, December 30, 1913.)

IN a recent number of the Transactions Sheffer $\ddagger$ presented an elegant and concise set of five postulates for Boolean algebras, and proved them mutually consistent and independent. Professor E. H. Moore§ has suggested a further interesting problem in connection with such sets of postulates, namely the determination of all general implicational relations

* Loc. cit., p. 268 (10).

† Loc. cit., p. 282 (2).

$\ddagger$ H. M. Sheffer, "A set of five postulates for Boolean algebras with application to logical constants," Transactions, vol. 14 (1913), pp. 481-488.

$\S$ E. H. Moore, "Introduction to a form of general analysis," New Haven Mathematical Colloquium, Yale University Press, page 82. 